\title{
A New Approach to Computation of Curvature Scale Space Image for Shape Similarity Retrieval
}

\author{
Farzin Mokhtarian, Sadegh Abbasi and Josef Kittler \\ Centre for Vision Speech and Signal Processing \\ Department of Electronic \& Electrical Engineering, \\ University of Surrey \\ Guildford, GU2 5XH \\ England \\ Email F.Mokhtarian@ee.surrey.ac.uk \\ Tel +44-1483-300800 extension 2288 \\ Fax +44-1483-534139
}

\begin{abstract}
The Curvature Scale Space image is a multi-scale organisation of the inflection points of a closed planar curve as it is smoothed. It consists of several arch shape contours, each related to a concavity or a convexity of the curve. In our recent works, we have used the maxima of these contours as shape descriptors to find similar shapes in large image databases. In this paper, we address the problem of shallow concavities. They may give rise to large contours in the CSS image. These contours may then match contours corresponding to deep and wide concavities during the matching process.

We have introduced a method to adjust the height of every CSS image contour which will be less than the height of the actual contour in the CSS image. A new global parameter for closed curves is also introduced. This parameter, like the CSS image representation, is robust with respect to noise, scale and change in orientation.
\end{abstract}

\section{Introduction}

The theory of the Curvature Scale Space representation of planar curves was described in [1] and since then has been used in object recognition [2], shape similarity retrieval ${ }^{1}[3]$ [4] and leaf classification [5]. In all applications, the maxima of the CSS contours have been used to represent the boundary of an object.

In the object recognition application, the ambiguities of CSS matching were resolved by a verification stage where the actual distance between the image and model curve is determined and compared.

In shape similarity retrieval, the user points to an image and wishes the system to find the most similar images to the input from the database. The user then verifies the outputs of the system and picks his/her desired images. Therefore, the above mentioned verification stage may be omitted in this application and the results of the CSS matching may appear as the output of the system.

${ }^{1}$ A demo of this work is available at the following web site: http://www.ee.surrey.ac.uk /Research/VSSP/imagedb/demo.html 
To construct the CSS image of a digital curve, its curvature zero_crossing points should be determined at different levels of smoothing. There will be two curvature zero_crossings on every concavity of the shape and as the curve becomes smoother these points approach each other and create a contour in the CSS image of the shape. When the concavity is filled, the two points join and represent the maximum of the relevant contour. The height of this contour then reflects the depth and size of the concavity. The deeper and larger the concavity, the higher the maximum.

If the shape contains a shallow concavity, its curvature zero_crossing points will not disappear immediately as the level of smoothing increases. The corresponding contour on the CSS image can then be a very large one. As a result, in shape similarity retrieval, an object with straight line segments may be matched to an object with a curved outline.

To solve this problem, in [3] and [4] two global parameters, eccentricity and circularity, were also used in conjunction with the maxima of the CSS image to represent the boundaries of objects. If the eccentricity or circularity of a model did not fall within a certain interval of the input ones, the model was rejected and CSS matching was not applied. We shall argue in this paper about even better results can be achieved by examining the maxima of the CSS image and reducing them to appropriate levels if the relevant segments are shallow concavities. The details of this method are described in Section 3.

On a database of 1100 images of marine animals, we tested the proposed method to find similar shapes from the database. Every image contained an object on a simple uniform background. After extracting the boundary of every object, it was sampled by 200 equally distant points and then the CSS image of the boundary was constructed. Using the the CSS image and the smoothed curves at different levels, the new maxima for the CSS image were calculated and stored. Every object was then represented by the new maxima of its CSS image. The image query consisted of pointing to one of the images of the database and asking the system to find images similar to the input.

The results affirmed a significant improvement in the performance of the system for the shapes with shallow concavities.

The following is the organisation of this paper. Section 2 is devoted to the concept of the CSS image. The problem of shallow concavities in CSS image is also explained in this section. Section 3 is devoted to the new method. A new global parameter for closed curves is also introduced in this section. In Section 4 the evaluation of the method together with the results is presented.

\section{Curvature Scale Space image}

Consider a parametric vector equation for a continuous curve $\Gamma$ :

$$
\boldsymbol{\Gamma}(s)=(x(s), y(s))
$$


where $s$ is the arc length of the boundary traversed. The formula for computing the curvature function can be expressed as:

$$
\kappa(s)=\frac{\dot{x}(s) \ddot{y}(s)-\ddot{x}(s) \dot{y}(s)}{\left(\dot{x}^{2}(s)+\dot{y}^{2}(s)\right)^{3 / 2}} .
$$

If $g(u, \sigma)$ is a 1-D Gaussian kernel of width $\sigma$, then $X(u, \sigma)$ and $Y(u, \sigma)$ represent the components of evolved curves [1],

$$
\begin{aligned}
& X(u, \sigma)=x(u) * g(u, \sigma) \\
& Y(u, \sigma)=y(u) * g(u, \sigma)
\end{aligned}
$$

where $*$ denotes convolution. According to the properties of convolution, the derivatives of every component can be calculated easily :

$$
\begin{aligned}
X_{u}(u, \sigma) & =x(u) * g_{u}(u, \sigma) \\
X_{u u}(u, \sigma) & =x(u) * g_{u u}(u, \sigma)
\end{aligned}
$$

and we will have similar formulas for $Y_{u}(u, \sigma)$ and $Y_{u u}(u, \sigma)$. Since the exact forms of $g_{u}(u, \sigma)$ and $g_{u u}(u, \sigma)$ are known, the curvature of an evolved digital curve can be computed easily:

$$
\kappa(u, \sigma)=\frac{X_{u}(u, \sigma) Y_{u u}(u, \sigma)-X_{u u}(u, \sigma) Y_{u}(u, \sigma)}{\left(X_{u}(u, \sigma)^{2}+Y_{u}(u, \sigma)^{2}\right)^{3 / 2}}
$$

\subsection{Curvature Scale'Space image and CSS matching}

The implicit function defined by $\kappa(u, \sigma)=0$, is the curvature scale space image of $\Gamma$. In digital form, it is a binary image and as shown in the right part of Figure 1, black points in this image are the locations of curvature zero_crossings during the process of evolution. The intersection of every horizontal line with the contours in this image indicates the locations of curvature zero crossings on the corresponding evolved curve $\Gamma_{\sigma}$.

The small contours of the CSS image represent the minor ripples on the boundary of object and can be ignored. Every boundary then is represented by the locations of the major maxima of its CSS image contours.

The matching algorithm which compares two sets of maxima from two different objects is presented in [3]. This algorithm assigns a match value as the measure of similarity between the two objects.

\subsection{The problem of shallow concavities}

In this section we demonstrate how two different types of concavities may create the same contours in the CSS image. An example is shown in Figure 1a. The boundary contains several shallow concavities and straight line segments. After removing the noise, two curvature zero_crossings remain for every concavity. For 


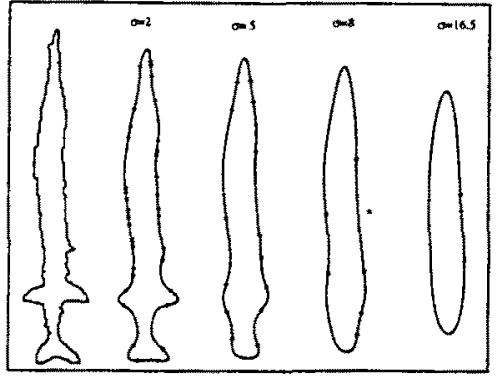

(a)

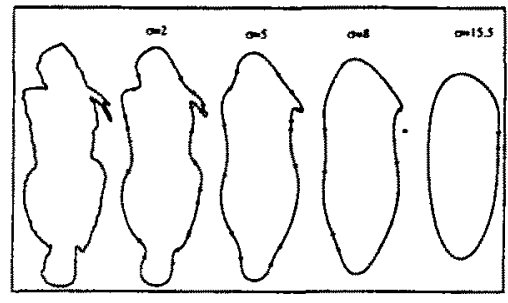

(c)

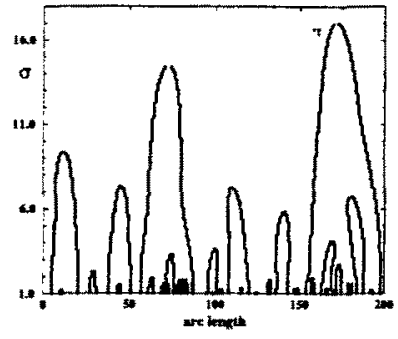

(b)

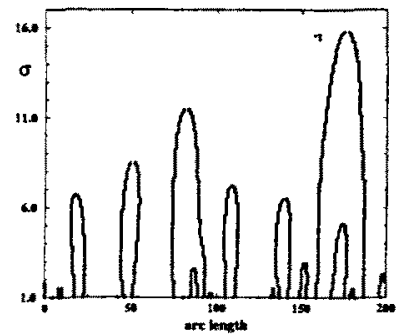

(d)

Fig. 1. Shallow and curved concavities, both create large contours on the CSS image. The marked contours relate to the marked concavities.

$\sigma=8$, the marked concavity is almost filled. The relevant zero crossings should disappear soon after this stage, but this actually happens at $\sigma=16.58$ and creates the largest maximum of the CSS image in Figure 1b.

In Figure 1c, a shape with curved segments is presented. For this shape, the segment between the two curvature zero_crossings of a concavity is not converted to a straight line until the final stages of smoothing. For $\sigma=8$, the marked concavity is still curved. It is filled at $\sigma=15.54$, and creates the largest maximum.

It can be shown that the rate of movement of curve points as sigma increases is almost proportional to the value of curvature at that point. Therefore, the movement of points, including zero_crossings, on straight line segments can be very small due to very low values of curvature. The relevant contour in the CSS image then can be very large as a result.

Considering the two objects and their CSS image representations in Figure 1, it is observed that although the boundaries are not similar, the two CSS images are almost similar. The output of the system, in response to the top object as the input, is shown in Figure 2c. The bottom object of Figure 1 is selected as the third most similar shape to the input shape. Other outputs are also dissimilar to the input. Note that we have not used our conventional global parameters, eccentricity and circularity, in this example. 


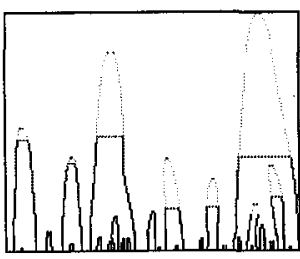

(a)

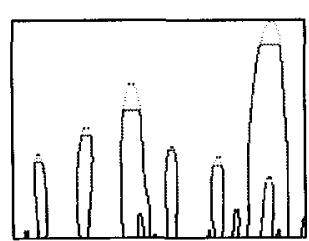

(b)

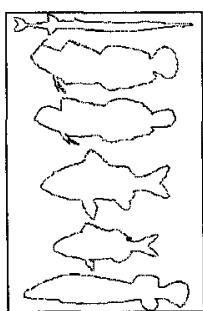

(c)

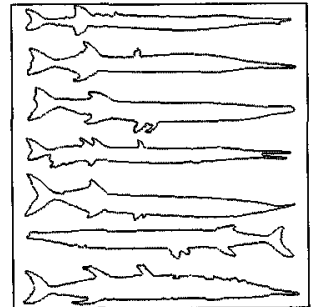

(d)

Fig. 2. a) The new maxima are significantly different for shallow concavities. b) No considerable changes for curved concavities. c) Result for a query using actual maxima. d) Results for the same query using new method.

\section{The proposed method}

The basic idea of our method is to select a new maximum for each CSS contour. For every contour, the new maximum is lower than the actual one. The difference depends on the shape of the concavity. For shallow concavities the difference will be large, whereas for curved concavities it will be negligible.

We can summarise our algorithm for locating the new maximum of a contour of the CSS image as follows:

1. Determine the maximum of the largest contour.

2. For the next lower scale, find the location of zero_crossings by following the two branches of the contour.

3. For the current level of smoothing, find the absolute value of the curvature at every point between the two zero_crossings and determine the maximum curvature in this segment.

4. If the maximum curvature is above a certain threshold, set the new maximum as the middle point of this segment, otherwise go to Step 2.

5. Exit if there are no maximum left, otherwise determine the next largest maximum of the CSS image and repeat Steps 2 to 5.

Figure $2 \mathrm{a}$ and $2 \mathrm{~b}$ show the new maxima of the CSS images of Figure 1. Note that the sets of maxima are not very similar. Figure $2 \mathrm{~d}$ shows the result of the same query as Figure $2 \mathrm{c}$ using the new set of maxima.

Normalisation, The absolute value of curvature is not robust with respect to scale. Therefore, we have to normalise the perimeter of the boundaries so that all boundaries have identical perimeter. consider $S_{i}$ as $S_{i}=\frac{P_{a v}}{P_{i}}$, where $S_{i}$ is the Scale factor for the $i$ th object and $P_{i}$ and $P_{a v}$ refer to the $i$ th object and the 
average perimeter respectively. To obtain a new perimeter for the $i$ th object, we multiply the $x$ and $y$ coordinates of its boundary points by $S_{i}$.

A new global parameter, We introduce a new global parameter for a closed planar curve as $\alpha=\sum_{i=1}^{n} d_{i}$, where $d_{i}$ is the difference between the old and new maximum of the $i$ th contour of the CSS image of the curve. The parameter will be near zero for curved shapes and will be large for shapes with shallow concavities. We have used this parameter for indexing to narrow down the range of searching.

\section{Evaluation and results}

To evaluate the method, we have created a small classified database which is a sub-set of our large database. There are 17 classes in this database, each consisting of about 8 objects. The procedure of evaluating and marking the performance of the system is as follows:

- Choose one of the objects in class one as the input query, and determine the first $n$ outputs of the system. These are the most similar images of the database to the input according to the system. $n=15$ is chosen for this test.

- Count the number of outputs which are in the same class as the input and let the result be a measure of performance of the system for that particular object.

- Repeat the previous steps to determine the performance of the system for class one, by summing up the performance measure of all members of this class.

- Determine the performance measures of all classes, repeating the above steps.

We first tested the proposed method on a database of 1100 images of marine animals. Each image consisted of just one object on a uniform background. The system software was developed using the $\mathrm{C}$ language under Unix operating system. The response rate of the system was less than two seconds for every user query. Generally, a significant improvement was observed for the shapes with shallow concavities. While the results for curved shapes remained almost unchanged and as good as before.

We also applied the above mentioned test to evaluate the performance of the new method. Several classes are represented in Figure 3. The results of the test for these classes are in Table 1. As this table shows, for group $c$, both methods show a $100 \%$ result. It means that whenever a member of this class was the input, all other members appeared in the first 15 outputs of the system. This figure for class $b$ is $28.5 \%$ with the previous method and $90 \%$ with the new method. On average, $90 \%$ of the members of this class were among the first 15 output of the system when one of the members has been the input.

Considering Table 1 and Figure 3, we realise that the performance of the system remains almost unchanged for curved shapes and improves significantly for shapes with shallow concavities. 


\begin{tabular}{|c|c|c|c|}
\hline Groups & $\begin{array}{c}\text { Previous } \\
\text { method } \\
\%\end{array}$ & $\begin{array}{c}\text { New } \\
\text { method } \\
\%\end{array}$ & Difference \\
\hline a & 31 & 62 & +31 \\
\hline b & 28.5 & 90 & +61.5 \\
\hline c & 100 & 100 & 0 \\
\hline d & 84 & 80 & -4 \\
\hline e & 86 & 87.5 & +1.5 \\
\hline f & 100 & 100 & 0 \\
\hline g & 100 & 100 & 0 \\
\hline h & 92 & 95 & +3 \\
\hline i & 78 & 78 & 0 \\
\hline
\end{tabular}

Table 1. Results of evaluation for different groups of figure 3.

\section{Conclusions}

This paper described a method to solve the problem of shallow concavities in Curvature Scale Space representation of planar curves. A new global parameter which distinguishes between curved shapes and shapes with straight line segments was also introduced. The method was tested on a database of 1100 images of marine creatures. It was then tested on a small classified database to obtain an objective evaluation of its performance. The results showed a major improvement in performance over CSS matching without the proposed CSS image maxima modification. The improvement was particularly significant for shapes with shallow concavities.

Acknowledgements Sadegh Abbasi is on leave from the University of Guilan, Rasht, Iran. He is grateful to the Ministry of Culture and Higher Education of Iran for its financial support during his research studies.

\section{References}

1. Mokhtarian, F. and Mackworth, A. K. "A theory of multiscale, curvature-based shape representation for planar curves" in PAMI 1992, VOL. 14, pp789-805.

2. Mokhtarian, F. "Silhouette-based isolated object recognition through curvature scale space" in IEEE Trans. PAMI 1995, Vol.17, pp.539-544

3. Mokhtarian, F. Abbasi S. and Kittler J. "Robust and Efficient Shape Indexing through Curvature Scale Space" in Proc. BMVC'96. Edinburgh, September 1996, pp 53-62.

4. Mokhtarian, F. Abbasi S. and Kittler J. "Efficient and Robust Retrieval by Shape Content through Curvature Scale Space" in Proc. of the First International Workshop on Image Databases and Multimedia Search, Amsterdam, Aug 1996, pp 35-42.

5. Abbasi S., Mokhtarian, F. and Kittler J. "Reliable Classification of Chrysanthemum Leaves through Curvature Scale Space" in the proc. of the Scale-Space '97 Conference, July 1997, Utrecht, Netherlands. 


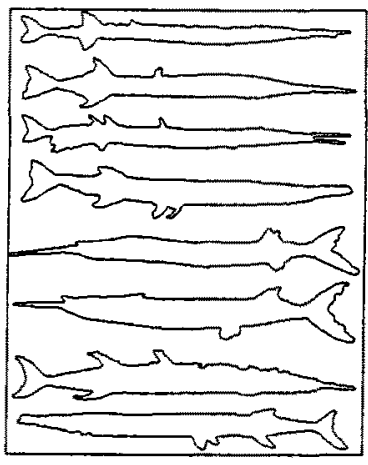

(a)

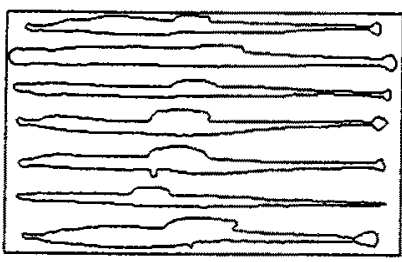

(b)

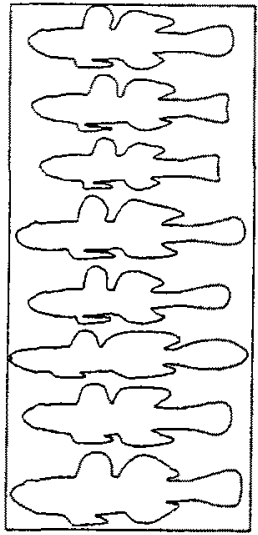

(c)

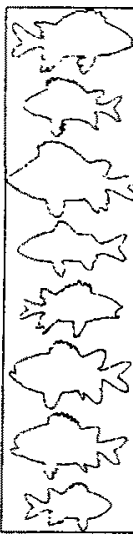

(d)

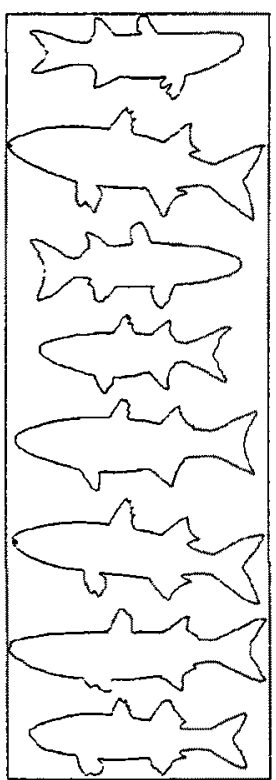

(e)

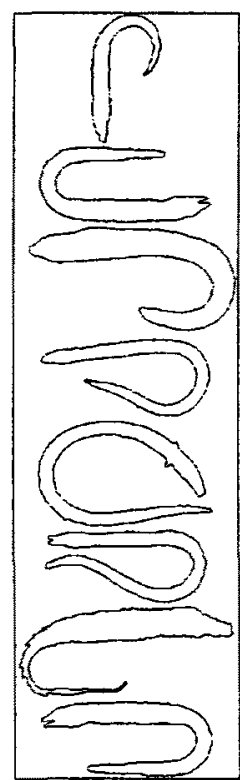

(f)

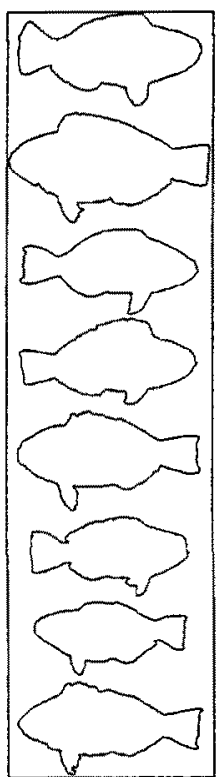

(g)

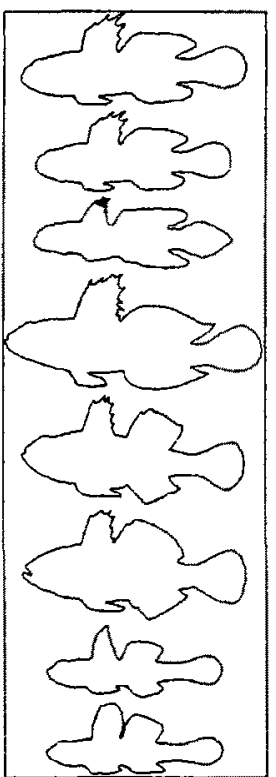

(h)

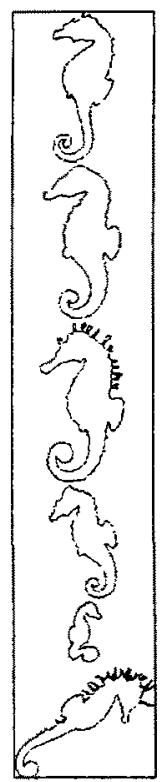

(i)

Fig. 3. Several classes of shapes used for testing the methods. The results for each class are given in Table 1 of Section 4. 